\title{
Postoperative pain
}

\author{
R. E. S. BULlinghAM \\ M.A., M.B., B.S., F.F.A.R.C.S.E. \\ Nuffield Department of Anaesthetics, Radcliffe Infirmary, Oxford OX2 $6 H E$
}

\section{Pain intensity after surgery}

The degree of pain reported by patients after surgery is highly variable (McQuay et al., 1982). Averaging across patients and operations typically shows one-third do not complain of postoperative pain, one-third have mild to moderate pain, and onethird have moderate to severe pain (Pflug and Bonica, 1977). The likelihood of severe pain is increased by certain factors (Loan and Morrison, 1967; Pflug and Bonica, 1977), of which the site of operation (thoracic $>$ upper abdominal $>$ lower abdominal $>$ superficial and peripheral operations) has been the most consistent association. Less consistent factors include type of anaesthetic and premedication, amount of anxiety, the amount and type of information given to the patient about the procedures, and the cultural and social background of the patient (Scott, Clum and Peoples, 1983). All these associations are of a very low statistical order and have no value in the prediction of postoperative pain intensity in the individual subject.

The biological basis of this variability probably originates in neurobiochemical differences which impart a varying capacity to modulate nociceptive inputs. Thus Terenius and Tamsen (1980) showed that patients with high preoperative endorphin levels in the cerebrospinal fluid require less pethidine for adequate pain relief after major abdominal surgery. A large number of other neurochemical substances also take part in nociception (Bullingham, 1984a), and presumably will show similarly variable responses during acute pain. Assuming these differences are genetically based and can vary independently of each other, a frequency distribution for the affected characteristic is expected which is humpshaped although not necessarily symmetrical. With pain after surgery, moderate intensity is most frequently reported and treatments are generally pitched at this value. However, it is the extremes corresponding to almost complete and almost absent

Present address: 'Rivendell', Wroxton Lane, Horley, Banbury, Oxon OX15 6BD. modulation of nociception which spoil therapeutic endeavours.

Those patients with no or minimal postoperative pain require no or minimal treatment: in particular, prophylactic or mandatory analgesic interventions can only do them harm. Even in circumstances where premedication and anaesthesia were managed without analgesic drugs, about $5 \%$ of patients undergoing minor limb surgery never needed any analgesic postoperatively (McQuay et al., 1982); about 10\% more were managed with a single dose of oral or parenteral analgesic (unpublished). These proportions would probably have totalled over $50 \%$ with a more typical perioperative management (Loan and Morrison, 1967). Although prophylactic perineural local anaesthetic blockade is popular in this situation, these figures suggest bona fide benefit may be available to less than half of the patients so treated.

Less information is available about patients with very severe pain. About $15 \%$ of general surgical postoperative patients received no or minimal pain relief from 10 or $15 \mathrm{mg}$ of subcutaneous morphine (Lasagna and Beecher, 1954). Similarly, in patients allowed to self-administer opioid analgesics intravenously from a motorised syringe pump (on-demand analgesia), about $15 \%$ of patients can be calculated to need more than 1.5 times the usual clinical dose (Tamsen et al., 1982). The proportion of individuals at the upper extreme thus seems to be comparable to that in the lower. Such patients are at risk of undertreatment of their pain, especially with the usual fixed-dose, fixed-interval analgesic regimens. They constitute a proportion of that well-documented population of postoperative patients with inadequate pain relief.

\section{Time-course of pain intensity after surgery}

Although typical time histories can be described for the onset of pain, rate of decline and range of short-term intensity, there is considerable variation between patients. The onset of postoperative pain is customarily defined by the time at which a patient 
first requests pain relief. In minor orthopaedic surgery, after a standardised anaesthetic using drugs with minimal residual analgesic effect, approximately $50 \%$ of patients had requested analgesia within $1 \mathrm{hr}$ and $75 \%$ within $3 \mathrm{hr}$ of ending the operation (McQuay et al., 1982). The remaining patients were then distributed in a very long tail: $15 \%$ of the patients had not required analgesia in the initial $6 \mathrm{hr}$, and one-third of these never needed analgesia at any time. Premedicant and intraoperative analgesic drugs with residual effects postoperatively (e.g. the long-acting opioid buprenorphine), and analgesic techniques applied at the end of the operation (e.g. perineural blockade with local anaesthetics), would move a proportion of patients towards later onset times. Conversely, more major surgical procedures would counterbalance such effects by tending to shift patients towards an earlier onset. The resulting distribution of onset times is rarely characterised, but it is an important conceptual statistic for a given operative population. If additional perioperative analgesic interventions are contemplated, then rationally these can only benefit those patients with onset times shorter than the duration of the treatment. Moreover, the shifts within the onset time distribution resulting from such treatments represent one aspect of the therapeutic benefit.

The decay of pain intensity postoperatively is generally assessed by the rate of decline in the intermittent parenteral use of opioid drugs. This is a very imperfect measure because it is subject to uncontrolled influences such as drug availability, and assumes that complete pain relief is achieved. In addition, the possible development of tolerance is ignored. The necessity to relieve the patient's pain, however, leaves few alternatives. Patient controlled on-demand analgesia (Tamsen et al., 1982; Jacobs et al., 1981) is a significant advance in methodology. It largely removes the first two problems although that of tolerance remains. After major surgery, with intermittent administration, the mean amount of opioid administered halves every $12-24 \mathrm{hr}$ in the first 3 days, and residual pain after treatment is halved about every 2 days (Nayman, 1979). This suggests that a typical value for the half-life of ${ }^{4}$ decay of postoperative pain would be about 1 day. This general pattern may differ markedly for specific types of operation and with individual patients. After intraabdominal procedures an almost constant consumption of narcotic is seen for the first 3 postoperative days before a decline occurs (Nayman, 1979), and demand analgesia confirms this pattern (Tamsen $e t$ al., 1979).

In contrast, after open meniscectomy without subsequent intra-articular drainage, marked increases in analgesic requirement may be seen as an effusion builds up within the joint. Increasing de- mand for analgesia after an initial stable period more 3 usually suggests the presence of complications, e.g., hip dislocation occurring after prosthetic replace-. ment surgery. Although, on average, major surgery $\overrightarrow{\vec{B}}$ causes greater initial pain than minor surgery, many $\underset{\sigma}{\sigma}$ minor surgical patients have an initial pain intensity equal to that seen after major surgery. Postoperative $\overline{\bar{c}}$ pain may, therefore, need vigorous initial treatment $\vec{\mathbb{}}$ whatever the magnitude of the surgery. The rate of $\bigcirc$ spontaneous decline in pain is, however, usually os much more rapid after less extensive surgery.

Mean pain intensity values conceal not only the wide variations which exist between patients, but also $\bar{\omega}$ the short-term within-patient variations. The latter may be cyclical, as with colic or pain associated with breathing after thoracotomy or upper abdominal. surgery. It may relate to particular events, such as physiotherapy or nursing procedures, or can appear $\underset{ }{\checkmark}$ to be random. The all-or-none analgesia from regional nerve blockade covers both background pain $\stackrel{\infty}{\oplus}$ and exacerbations. In contrast, any technique with a graded dose-response must perforce be either insuffi- $\frac{}{J}$ cient at the peaks of pain intensity or overwhelming $\vec{b}$ at the troughs. In situations where there is marked $\mathbb{D}$ variation in pain intensity, blockade is the more $\Phi$ rational approach.

\section{Pain relief after surgery}

The degree of pain relief reported by patients afte treatment varies from none to complete. After general surgery, analgesia from $10-15 \mathrm{mg}$ of parenteral morphine is complete or almost complete in approximately one-third of the patients, moderate in a second third and minimal or none in the remainder $\triangle$ (Lasagna and Beecher, 1954).

The factors responsible for individual variation in 3 pain relief fall into two main categories: those related to the biological interaction of the treatment with the nociceptive mechanisms and those related to the $\overline{0}$ practical aspects of delivering the treatment. Treatments with a graded dose-effect relationship, such as analgesic drugs, are associated with factors in both categories, whereas treatments with an all-or-none effect, such as nerve blockade, are predominantly influenced by delivery.

Neurochemical differences are the probable basis of much of the biological component. In postoperative patients using demand analgesia, the plasma $N$ pethidine concentration when complete pain relief $\%$ was achieved had a significant negative linear corre- $N$ lation with preoperative cerebrospinal fluid endor- N phin concentration (Terenius and Tamsen, 1980). This suggests that exogenous analgesic drugs supple- 0 ment endogenous analgesic neurochemicals. The $\overparen{\Phi}$ total required for complete relief-obtained by $\stackrel{\oplus}{+}$ summing these separate contributions-may be a 0 
relatively constant amount even for different patients. The usual regimen of administering a fixed dose of analgesic should then give complete relief in those patients in whom the sum of endogenous and exogenous analgesic reaches or exceeds a critical value.

Those with the highest endogenous component should have the least pain intensity before treatment and be the most likely to get complete pain relief with a fixed dose of drug. Thus, in the $4 \mathrm{hr}$ after $10 \mathrm{mg}$ of parenteral morphine, about $90 \%$ of postoperative patients with slight pain showed complete relief compared with about 60,45 , and $30 \%$ for moderate, severe and very severe initial intensities (Lasagna, 1962). Similarly, a good positive correlation was found between peak amount of pain relief and the initial severity of pain. This in part reflects that those with severe pain could move further along the scale of pain intensity before pain relief became total. As a corollary, if the usual predetermined fixed minimal doses are given, many patients with mild pain must receive more drug than is needed for complete relief and so be unnecessarily exposed to dose-related side effects. The analgesic effect seen from administering inactive medication (the placebo effect) probably also arises from the stimulation of endogenous antinociceptive mechanisms (Gracely et al., 1983), both opioid and non-opioid. The magnitude of the effect is large, being typically about $50 \%$ of the pain relief from $10 \mathrm{mg}$ of morphine. Although this implies that the biological background is capable of substantial alteration by non-specific mechanisms, there is presently insufficient information to utilise such manipulation deliberately or reliably within the individual patient. Some of the newer techniques such as transcutaneous electrical nerve stimulation may allow this in the future.

Causes of variability related to the delivery of analgesia are better understood and, in particular, can be influenced by clinical choices such as which technique and drugs are used. For neural blockade, the critical determinant of success is that there should be a simple, unmistakable, positive criterion for correct placement of the blocking agent. Where the procedure can be carried out under direct vision, as with cryoanalgesia of the ilio-inguinal nerve at herniorraphy (Wood et al., 1981); or where some characteristic event occurs, such as cerebrospinal fluid flow after dural puncture during spinal analgesia, the rate of failure is close to zero. With less sharp end-points, there is more chance of incorrect placement and an appreciably higher failure rate. Blind blockade procedures which rely solely on average anatomy and proportions can have failure rates approaching $30-40 \%$ for each nerve blocked. Perioperative peripheral nerve blocks with bupivacaine have become popular for the provision of postopera- tive analgesia but, as in the foot, commonly involve several separate nerves, each of which is capable of independent anatomical variations. Complete blockade of every nerve is then unlikely, but equally so is the risk of complete failure. Although the chance is high for at least a partial effect with some reduction in the requirement for other analgesics, the individual outcome is unpredictable. With analgesic drugs, a significant source of variability arises from the need to maintain adequate amounts of drug at the site of action. Thus, morphine or pethidine given in intermittent intramuscular doses are absorbed at very variable rates, even within the same patient (Mather, 1983). The resulting plasma drug concentrations are in many cases insufficient to give effective analgesia for at least part of the dose interval, and the dose-todose variability causes analgesia to be intermittent and unpredictable. Occasional intramuscular doses are so slowly available systemically that, in effect, a complete failure of drug administration occurs. Presumably, this is because of unintentional injection of the drug into poorly perfused tissues such as fat or fascia. Similar variability may be anticipated for subcutaneous administration. With non-parenteral routes, there may be even greater variability if extensive metabolism takes place before the systemic circulation is reached. The high and variable hepatic first pass effect of most opioid drugs makes the oral and rectal routes of little practical use in the treatment of postoperative pain. Only the intravenous route can ensure certainty of systemic drug delivery and remove the effects of dose-to-dose variation within the patient. There still remain interindividual differences in drug distribution and elimination, which for opioid analgesics are typically still capable of giving 5 -fold differences in body drug concentration for a given intravenous dose.

\section{Time-course of pain relief after surgery}

The time course of the provision of pain relief after surgery is usually assumed to resemble the natural time course that pain intensity would have if pain were untreated. However, certain forms of treatment may alter circumstances such that this is not so. The effect of delaying the onset time of postoperative pain and of the development of tolerance are examples considered here.

In patients undergoing inguinal herniorraphy, paravertebral blockade with bupivacaine significantly reduced the requirement for additional analgesic therapy in the first $24 \mathrm{hr}$ postoperatively in comparison with an untreated group. However, analgesic use was also significantly lower over the following 3 days, well outside the duration of action of the block (Wood et al., 1981). This supports the widely held but little studied clinical view that 
delaying the onset of postoperative pain can reduce subsequent pain. It is not known whether this is a genuine biological response and pain intensity is actually less after the delay, or whether it represents a psychological difference related to exposure to different maximum pain intensities.

Equivalent groups of patients undergoing total prosthetic hip replacement were managed perioperatively either with balanced anaesthesia using $10 \mathrm{mg}$ of intravenous methadone as the analgesic component, or by extradural local anaesthetic blockade intraoperatively followed by a mandatory intravenous dose of $10 \mathrm{mg}$ of methadone in the recovery period $3 \mathrm{hr}$ after induction of anaesthesia. Both groups were given on-demand analgesia with methadone for subsequent analgesic supplementation. Despite the later timing of the methadone dose, the latter group demanded significantly more methadone and correspondingly achieved significantly higher plasma methadone concentrations (Porter et al., 1983). It was suggested that tolerance had acutely developed to the postoperative narcotic bolus. Similar acute narcotic tolerance to analgesic, respiratory and hormonal effects can be produced by the administration of large intravenous boluses before the start of surgery (McQuay, Bullingham and Moore, 1981), or in animals. The critical determinant for the development of tolerance seems to be the presence of opioid drug in excess of that required to balance the nociceptive input. Although there appears to be no serious clinical sequelae when predetermined doses of opioids are used, acute tolerance may be important both as a safety mechanism when such doses are excessive and as a source of inter-patient variability.

\section{Aims and strategies for treatment}

The final aim of therapy could be seen as the complete relief of postoperative pain, throughout the whole postoperative period, with no treatment related side effects. This might be achieved, for example, by some ideal drug whose only effect was dose-related analgesia and which was infused at a constant supramaximal rate over a suitable length of time. Such supramaximal analgesia would in practice have surgical drawbacks: many complications including infection, venous thrombosis and infarction have pain as an early symptom, and for many operations pain may usefully limit the functional strain put on the operated area until adequate healing has occurred. Moreover, development of tolerance may be an added risk where administered drug is above that needed for full analgesic effect. Ideally, therefore, an exact balance between pain at rest in the operative site and pain relief would be better. This implies the need for a properly regulated reduction in analgesic effect. In reality, all current 3 therapies have unwanted effects and patients may $\stackrel{\square}{\square}$ settle for less than complete relief if side effects are $\subseteq$ reduced significantly. The present aim for treatment $\overrightarrow{\vec{c}}$ may be regarded as the time-dependent maximisa- $-\frac{?}{0}$ tion of comfort (Jacobs et al., 1981). Comfort is the? resultant of adding all the wanted effects of therapy, $\frac{\bar{c}}{\bar{s}}$ which could include pain relief, euphoria, and $\vec{\sigma}$ sedation (in some patients) and subtracting all the $\bigcirc$ unwanted effects, which could include nausea and vomiting, dysphoria, and sedation (in some patients). $\overrightarrow{0}$ It should be noted that only the individual subject can perform this judgement: some factors like seda- $\vec{\omega}$ tion may be seen as desirable by one patient and undesirable by another. Pre-emptive and mandatory $\frac{0}{3}$ analgesic therapies have the disadvantage of remov- 3 ing this opportunity for choice. For example, somes patients given certain local anaesthetic blocks might have preferred other methods of analgesia where $\vec{\circ}$ numbness and muscular paralysis were not concomitants. Other consequences not directly detectable by the patient, such as stiffness resulting from inability to move, respiratory depression and tolerance, may $\overrightarrow{ }$ impose clinical limitations on the allowed choice, $\mathbb{\Phi}$ duration and intensity of therapy.

No current therapeutic strategy meets all these $\frac{2}{3}$ requirements. Patient-controlled on-demand intravenous administration of opioid analgesia comes cloz্,, $\vec{\bullet}$ but dosage limitations are still usually imposed in the absence of good measures of respiratory function and expensive special equipment is needed. The technique is an invaluable research tool for postoperative pain. The usual intermittent intramuscular administration of analgesic drug is a form of ondemand analgesia, but even at best the time-scale of $\mathscr{\mathbb { Q }}$ dosing and the size of the dose are rarely well $\overrightarrow{\overrightarrow{0}}$ matched to the biological processes. In practice, these 3 factors are ordinarily overshadowed by the failure to $?$ make drug readily available to patients as and when required. Patient control is the key feature. All too often intramuscular drug administration can occur only at set times. If a controlled drug is used, it involves a discouraging number of administrative $\frac{3}{6}$ procedures. These may worsen disproportionately at $\delta$ night-time, and may be dominated by misinformed $₹$ nursing attitudes towards narcotics and pain. Perhaps the most significant practical feature of the introduc- $D$ tion of non-controlled parenteral analgesic drugs, such as meptazinol and the mixed agonist-antagonist $\bar{N}$ opioids, is the potential improvement in the availabil- $\Omega$ ity of strong analgesics to patients. Sublingual $N$ administration of buprenorphine (Drug and Thera- N peutics Bulletin, 1982) is similarly important in extending the availability of strong analgesics beyond 0 the usual predetermined $1-2$ days of parenteral dosing. All the non-parenteral analgesics which can $\stackrel{f}{?}$ be used as alternatives to parenteral opioids (e.g. the 
non-steroidal anti-inflammatory analgesics and paracetamol) have a limited maximal effect equivalent at most to $10-15 \mathrm{mg}$ of parenteral morphine (Bullingham, 1984b). This may be insufficient in many patients if used too soon or too arbitrarily as a replacement for parenteral opioids.

Regional analgesic procedures are technically demanding and have often been accepted uncritically without controlled studies. After upper abdominal surgery, intermittent intravenous pethidine titrated to complete pain relief gives analgesia and respiratory function just as good as that from extradural bupivacaine (Muneyuki et al., 1968). Although the duration of peripheral perineural blockade with bupivacaine is $12 \mathrm{hr}$ or more, the duration of action of local anaesthetics given spinally (i.e. extradurally or intrathecally) is only about $2 \mathrm{hr}$. Spinal administration of narcotics can also give analgesia lasting $12 \mathrm{hr}$ or more, without effects on other sensory modalities or motor function (Bullingham, McQuay and Moore, 1982). However, this technique cannot be recommended for routine clinical use until a more detailed understanding of its mode of action and unexpected side effects is available (Bullingham, McQuay and Moore, 1983).

\section{Conclusion}

Although the biological background to postoperative pain does not change, the extent of knowledge about it does. Progress in the neurobiochemistry of nociceptive mechanisms should broaden old therapeutic avenues and eventually yield new ones. It is, however, unlikely to help immediately the clinical treatment of postoperative pain. Clinical investigation is still needed to sharpen wisdom and settle scepticism by defining more closely such phenomena as tolerance and the placebo reaction, and by objectively comparing methods and regimens. In the last analysis, however, the principal problem is not lack of understanding, but lack of application. The key concept is the unpredictable variability of postoperative pain. The key consequence is unfailing belief in the report of the patient; while the key conduct is to individualise the delivery of relief. Adequate drugs and techniques are available to the practitioner. The essential need is for organisation and enthusiasm in order to make them available to the patient.

\section{References}

Bullingham, R.E.S. (1984a) Physiological mechanisms in pain. In: Acute Pain. (Eds. G. Smith and B. G. Covino), Vol. 1. International Medical Review Series in Anaesthesiology, Butterworth Scientific, London (in press).
Bullingham, R.E.S. (1984b) Synthetic analgesics. In: Recent Advances in Anaesthesia and Analgesia 15, (Eds. R. S. Atkinson and A. P. Adams). Churchill-Livingstone, Edinburgh (in press).

BUllingham, R.E.S., MCQUAY, H.J. \& MOORE, R.A. (1982) Extradural and intrathecal narcotics. In: Recent Advances in Anaesthesia and Analgesia 14, (Eds. R. S. Atkinson and C. Langton-Hewer), p. 141. Churchill-Livingstone, Edinburgh.

BUllingham, R.E.S., MCQUAY, H.J. \& MOORE, R.A. (1983) Principles of use of extradural and intrathecal narcotics. In: Anaesthesia Review 2, (Ed. L. Kaufman), p. 137. ChurchillLivingstone, Edinburgh.

DRUG AND THERAPEUTICS BULLETIN (1982) Sublingual buprenorphine, $20,74$.

Gracely, R.H., Dubner, R., Wolskee, P.J. \& Deeter, W.R. (1983) Placebo and naloxone can alter post-surgical pain by separate mechanisms. Nature, 306, 264.

JACOBS, O.L.R., BullinghaM, R.E.S., DAVIES, W.L. \& REASBECK, M.P. (1981) Feedback control of postoperative pain. IEEE Conference Publication Number 194, Control and its applications, 52-56.

LASAGNA, L. (1962) The psychophysics of clinical pain. Lancet, ii, 572.

LASAGNA, L. \& BeEcheR, H.K. (1954) The optimal dose of morphine. Journal of the American Medical Association, 156, 230.

LOAN, W.B. \& MORRISON, J.D. (1967) The incidence and severity of postoperative pain. British Journal of Anaesthesia, 39, 695.

MATHER, L.E. (1983) Pharmacokinetic and pharmacodynamic factors influencing the choice, dose and route of administration of opiates for acute pain. In: Opiate Analgesia. (Ed. R. E. S. Bullingham), Clinics in Anaesthesiology, Volume 1, p. 17. W. B. Saunders: London.

MCQUAY, H.J., BUllingham, R.E.S. \& MOORE, R.A. (1981) Acute opiate tolerance in man. Life Sciences, 28, 2513.

MCQuaY, H.J., BUllingham, R.E.S., MoORE, R.A., Evans, P.J.D. \& LLOYD, J.W. (1982) Some patients don't need analgesics after surgery. Journal of the Royal Society of Medicine, 75, 705.

MUNEYUKI, M., UeDA, Y., URABE, N., TAKeShita, H. \& INomoto, A. (1968) Postoperative pain relief and respiratory function in man: comparison between intermittent intravenous injections of meperidine and continuous lumbar epidural analgesia. Anaethesiology, 29, 304.

NAYMAN, J. (1979) Measurement and control of postoperative pain. Annals of the Royal College of Surgeons of England, 61, 420.

Pflug, A.E. \& BonICA, J.J. (1977) Physiopathology and control of postoperative pain. Archives of Surgery, 112, 773.

PorTer, E.J.B., MCQuay, H.J., Bullingham, R.E.S., WeIR, L., ALLEN, M.C. \& MOORE, R.A. (1983) Comparison of intraoperative and postoperative methadone: acute tolerance to the postoperative dose? British Journal of Anaesthesia, 55, 325.

ScotT, L.E., Clum, G.A. \& Peoples, J.B. (1983) Preoperative predictors of postoperative pain. Pain, 15, 283.

Tamsen, A., Hartvig, P., Dahlstrom, B., Lindstrom, B. \& HolmDAHL, M.H. (1979) Patient controlled analgesic therapy in the early postoperative period. Acta Anaesthesiologica Scandinavica, 23, 462.

Tamsen, A., Hartvig, P., Fagerlund, C., Dahlstrom, B. \& BONDESSON, U. (1982) Patient-controlled analgesic therapy: clinical experience. Acta Anaesthesiologica Scandinavica, 74, (Suppl), 157.

TEREniUs, L. \& TAMSEN, A. (1982) Endorphins and the modulation of acute pain. Acta Anaesthesiologica Scandinavica, 74, (Suppl), 21

WOOD, G.J., LLOYD, J.W., BUlLinghaM, R.E.S., BRITTON, B.J. \& FINCH, D.R.A. (1981) Postoperative analgesia for day-case herniorraphy. Anaesthesia, 36, 603. 